Bull. Korean Math. Soc. 50 (2013), No. 3, pp. 1049-1060

http://dx.doi.org/10.4134/BKMS.2013.50.3.1049

\title{
HILBERT 2-CLASS FIELD TOWERS OF IMAGINARY QUADRATIC FUNCTION FIELDS
}

\author{
HWANYUP JUNG
}

\begin{abstract}
In this paper we study the infiniteness of Hilbert 2-class field towers of imaginary quadratic function fields over $\mathbb{F}_{q}(T)$, where $q$ is a power of an odd prime number.
\end{abstract}

\section{Introduction and statement of the results}

For a number field $F$, let $F_{0}^{(2)}=F$ and $F_{n+1}^{(2)}$ be the Hilbert 2-class field of $F_{n}^{(2)}$ for $n \geq 0$. Then the sequence of fields

$$
F=F_{0}^{(2)} \subset F_{1}^{(2)} \subset \cdots \subset F_{n}^{(2)} \subset \cdots
$$

is called the Hilbert 2-class field tower of $F$ and we say that $F$ has an infinite Hilbert 2-class field tower if $F_{n}^{(2)} \neq F_{n+1}^{(2)}$ for all $n \geq 0$. Assume that $F$ is an imaginary quadratic number field. Let $r_{2}\left(\mathrm{Cl}_{F}\right)$ denote the 2-rank of class group $\mathcal{C} l_{F}$ of $F$. By Golod-Shafarevich's Theorem, $F$ has an infinite Hilbert 2-class field tower if $r_{2}\left(\mathrm{Cl}_{F}\right) \geq 5$. It has been conjectured by Martinet [6] that the Hilbert 2-class field tower of $F$ is infinite if $r_{2}\left(\mathrm{Cl}_{F}\right) \geq 4$. Let $r_{4}\left(\mathrm{Cl}_{F}\right)=r_{2}\left(\mathrm{Cl}_{F}^{2}\right)$ be the 4-rank of $\mathrm{Cl}_{F}$. It has been shown by Koch [5] and Hajir [3, 4] that $F$ has an infinite Hilbert 2-class field tower if $r_{4}\left(\mathrm{Cl}_{F}\right) \geq 3$. In [2], Gerth has proved that a positive proportion of the imaginary quadratic number fields $F$ with $r_{2}\left(\mathcal{C l}_{F}\right)=r$ have infinite Hilbert 2-class field towers and $r_{4}\left(\mathcal{C l}_{F}\right)=s$ for $r=3,1 \leq s \leq 3$ and for $r=4,0 \leq s \leq 4$.

Let $k=\mathbb{F}_{q}(T)$ be a rational function field over the finite field $\mathbb{F}_{q}, \infty=(1 / T)$ and $\mathbb{A}=\mathbb{F}_{q}[T]$. For a finite separable extension $F$ of $k$, write $\mathcal{O}_{F}$ for the integral closure of $\mathbb{A}$ in $F$ and $H_{F}$ for the Hilbert class field of $F$ with respect to $\mathcal{O}_{F}$ (see [7]). Let $\ell$ be a prime number. Let $F_{0}^{(\ell)}=F$ and $F_{n+1}^{(\ell)}$ be the Hilbert $\ell$-class field of $F_{n}^{(\ell)}$ for $n \geq 0$, i.e., $F_{n+1}^{(\ell)}$ is the maximal extension of $F_{n}^{(\ell)}$ inside

Received July 7, 2012.

2010 Mathematics Subject Classification. 11R11, 11R58.

Key words and phrases. Hilbert 2-class field tower, imaginary quadratic function field.

This work was supported by the National Research Foundation of Korea(NRF) grant funded by the Korea government(MEST)(No.2010-0008139). 
$H_{F_{n}^{(\ell)}}$ whose degree over $F_{n}^{(\ell)}$ is a power of $\ell$. The sequence of fields

$$
F=F_{0}^{(\ell)} \subset F_{1}^{(\ell)} \subset \cdots \subset F_{n}^{(\ell)} \subset \cdots
$$

is called the Hilbert $\ell$-class field tower of $F$. We say that the Hilbert $\ell$-class field tower of $F$ is infinite if $F_{n}^{(\ell)} \neq F_{n+1}^{(\ell)}$ for each $n \geq 0$. Let $\mathcal{C} l_{F}$ and $\mathcal{O}_{F}^{*}$ be the ideal class group and the group of units of $\mathcal{O}_{F}$, respectively. For any multiplicative abelian group $A$, write $r_{\ell}(A)=\operatorname{dim}_{\mathbb{F}_{\ell}}\left(A / A^{\ell}\right)$ for the $\ell$-rank of $A$. The following theorem is a function field analog of Golod-Shafarevich due to Schoof.

Theorem 1.1 (Schoof [9]). Let $F$ be a finite separable extension of $k$. Then the Hilbert $\ell$-class field tower of $F$ is infinite if

$$
r_{\ell}\left(\mathcal{C} l_{F}\right) \geq 2+2 \sqrt{r_{\ell}\left(\mathcal{O}_{F}^{*}\right)+1}
$$

Assume that $q$ is odd. Let $F$ be an imaginary quadratic function field over $k$, i.e., $F$ is a quadratic extension of $k$ in which $\infty$ is ramified. Fix a generator $\gamma$ of $\mathbb{F}_{q}^{*}$. Let $\mathcal{P}$ be the set of monic irreducible polynomials in $\mathbb{A}$. Then $F$ can be written as $F=k(\sqrt{D})$ with $D=\gamma^{a} P_{1} \cdots P_{t}, a \in\{0,1\}, P_{i} \in \mathcal{P}$ for $1 \leq i \leq t$ and $2 \nmid \operatorname{deg} D$. Here, $D$ is uniquely determined by $F$ and write $D_{F}=D$. Let $s_{F}$ be the number of monic irreducible divisors $P_{i}$ of $D_{F}$ of odd degree. Since $\operatorname{deg} D_{F}$ is odd, $s_{F}$ is a positive odd integer. We will assume that $\operatorname{deg} P_{i}$ is odd for $1 \leq i \leq s_{F}$ and $\operatorname{deg} P_{i}$ is even for $s_{F}+1 \leq i \leq t$. For $0 \neq N \in \mathbb{A}$, write $\omega(N)$ for the number of monic irreducible divisors of $N$. By genus theory ([1, Corollary 3.5]), we have $r_{2}\left(\mathcal{C} l_{F}\right)=\omega\left(D_{F}\right)-1$. Since $\mathcal{O}_{F}^{*}=\mathbb{F}_{q}^{*}$ and $r_{2}\left(\mathcal{O}_{F}^{*}\right)=1$, by Theorem 1.1, we see that $F$ has an infinite Hilbert 2-class field tower if $\omega\left(D_{F}\right) \geq 6$. The following theorem is a function field analogue of Koch and Hajir's one.

Theorem 1.2. Let $F$ be an imaginary quadratic function field over $k$. Let $s_{F}$ be the number of distinct monic irreducible divisors of $D_{F}$ of odd degree. If $r_{4}\left(\mathcal{C} l_{F}\right) \geq 3$, then the Hilbert 2-class field tower of $F$ is infinite, except the cases that $q \equiv 1 \bmod 4, \omega\left(D_{F}\right)=4$ and $s_{F}=3$. In this exceptional case, if $D_{F}$ has monic irreducible divisors $P$ and $Q$ such that $\operatorname{deg} P$ is divisible by 4 and $\left(\frac{P}{Q}\right)_{4}=1$, then the Hilbert 2 -class field tower of $F$ is infinite, where $\left(\frac{*}{*}\right)_{4}$ is the 4-th power residue symbol.

For any positive integers $n, r$ and integer $s$ with $0 \leq s \leq r$, let $X_{r ; n}$ be the set of imaginary quadratic function fields $F$ with $r_{2}\left(\mathcal{C} l_{F}\right)=r$ and $\operatorname{deg}\left(D_{F}\right)=n$, $X_{r, s ; n}$ be the subset of $X_{r ; n}$ consisting of $F \in X_{r ; n}$ with $r_{4}\left(\mathcal{C} l_{F}\right)=s$ and $X_{r, s ; n}^{*}$ be the subset of $X_{r, s ; n}$ consisting of $F \in X_{r, s ; n}$ having an infinite Hilbert 2-class field tower. We define a density $\delta_{r, s}^{*}$ by

$$
\delta_{r, s}^{*}=\liminf _{\substack{n \rightarrow \infty \\ n: \text { odd }}} \frac{\left|X_{r, s ; n}^{*}\right|}{\left|X_{r ; n}\right|} .
$$

Then we have: 
Theorem 1.3. $\delta_{3, s}^{*} \geq 2^{-9}$ for $1 \leq s \leq 3$ and $\delta_{4, s}^{*} \geq 2^{-14}$ for $0 \leq s \leq 4$.

Remark 1.4. Theorem 1.3 means that a positive proportion of imaginary quadratic function fields $F$ with $r_{2}\left(\mathcal{C} l_{F}\right)=r$ have infinite Hilbert 2-class field towers and $r_{4}\left(\mathcal{C} l_{F}\right)=s$ for $r=3,1 \leq s \leq 3$ and for $r=4,0 \leq s \leq 4$.

\section{Preliminaries}

\subsection{Martinet's inequality}

Let $E$ and $K$ be finite geometric separable extensions of $k$ such that $E / K$ is a cyclic extension of degree $\ell$ with $\Delta=\operatorname{Gal}(E / K)$, where $\ell$ is a prime number not dividing $q$. Let $\mathcal{O}_{E}$ be the integral closure of $\mathbb{A}$ in $E$ and $\mathcal{O}_{E}^{*}$ be the group of units of $\mathcal{O}_{E}$. Then $H^{0}\left(\Delta, \mathcal{O}_{E}^{*}\right)$ and $H^{1}\left(\Delta, \mathcal{O}_{E}^{*}\right)$ are elementary abelian $\ell$-groups with

$$
\frac{\left|H^{0}\left(\Delta, \mathcal{O}_{E}^{*}\right)\right|}{\left|H^{1}\left(\Delta, \mathcal{O}_{E}^{*}\right)\right|}=\ell^{-1} \prod_{\mathfrak{p}_{\infty} \in S_{\infty}(K)}\left|\Delta_{\mathfrak{p}_{\infty}}\right|,
$$

where $S_{\infty}(K)$ is the set of primes of $K$ lying above $\infty$ and $\Delta_{\mathfrak{p}_{\infty}}$ denotes the decomposition group of $\mathfrak{p}_{\infty}$ in $\Delta$. Following the arguments in $[6, \S 2]$, we get the following proposition.

Proposition 2.1. Let $E / K$ be as above. Let $\gamma_{E / K}$ be the number of prime ideals of $\mathcal{O}_{K}$ that ramify in $E$ and $\rho_{E / K}$ be the number of primes $\mathfrak{p}_{\infty}$ in $S_{\infty}(K)$ that ramify or inert in $E$. Then the Hilbert $\ell$-class field tower of $E$ is infinite if

$$
\gamma_{E / K} \geq\left|S_{\infty}(K)\right|-\rho_{E / K}+3+2 \sqrt{\ell\left|S_{\infty}(K)\right|+(1-\ell) \rho_{E / K}+1}
$$

The inequality (2.1) is called the Martinet's inequality. Now, by using Proposition 2.1, we give some sufficient conditions for an imaginary quadratic function field $F$ to have infinite Hilbert 2 -class field tower. Let $\left(\frac{*}{*}\right)$ denote the quadratic residue symbol in $\mathbb{A}$.

Corollary 2.2. Let $F$ be an imaginary quadratic function field over $k$. If there exists a nonconstant divisor $D^{\prime}$ of $D_{F}$ such that either $D^{\prime}$ or $D_{F} / D^{\prime}$ is monic of even degree and $\left(\frac{D^{\prime}}{P_{i}}\right)=1$ for monic irreducible divisors $P_{i}(1 \leq i \leq 4)$ of $D_{F}$, then $F$ has an infinite Hilbert 2-class field tower.

Proof. Let $K=k\left(\sqrt{D^{\prime}}\right)$ and $E=K F$. Since either $D^{\prime}$ or $D_{F} / D^{\prime}$ is monic of even degree, the infinite prime $\mathfrak{p}_{\infty}$ of $F$ splits in $E$. It is easy to see that any finite primes of $F$ is unramified in $E$. Hence $E$ is contained in $F_{1}^{(2)}$. Since $P_{1}, P_{2}, P_{3}$ and $P_{4}$ split in $K$, we have $\gamma_{E / K} \geq 8$. We also have $\left|S_{\infty}(K)\right|=$ $\rho_{E / K}=2$ or $\left(\left|S_{\infty}(K)\right|, \rho_{E / K}\right)=(1,0)$ according as $D^{\prime}$ is monic of even degree or $D_{F} / D^{\prime}$ is monic of even degree. By Proposition 2.1 on $E / K$, we see that $E$ has an infinite Hilbert 2-class field tower. Since $E$ is contained in $F_{1}^{(2)}, F$ also has an infinite Hilbert 2-class field tower. 
Corollary 2.3. Let $F$ be an imaginary quadratic function field over $k$. If $D_{F}$ has two distinct nonconstant monic divisors $D_{1}$ and $D_{2}$ of even degrees satisfying $\left(\frac{D_{1}}{P_{i}}\right)=\left(\frac{D_{2}}{P_{i}}\right)=1$ for monic irreducible divisors $P_{i}(i=1,2)$ of $D_{F}$, then $F$ has an infinite Hilbert 2-class field tower.

Proof. Let $K=k\left(\sqrt{D_{1}}, \sqrt{D_{2}}\right)$ and $E=K F$. Since $\infty$ splits completely in $K$, the infinite prime $\mathfrak{p}_{\infty}$ of $F$ splits in $E$. It is easy to see that any finite primes of $F$ is unramified in $E$. Hence $E$ is contained in $F_{1}^{(2)}$. By applying Proposition 2.1 on $E / K$ with $\gamma_{E / K} \geq 8$ and $\left|S_{\infty}(K)\right|=\rho_{E / K}=4$, we see that $E$ has an infinite Hilbert 2-class field tower. Since $E$ is contained in $F_{1}^{(2)}, F$ also has an infinite Hilbert 2-class field tower.

Corollary 2.4. Let $F$ be an imaginary quadratic function field over $k$. If $D_{F}$ has two distinct nonconstant monic divisors $D_{1}$ and $D_{2}$ of even degrees satisfying $\left(\frac{D_{1}}{P_{1}}\right)=\left(\frac{D_{2}}{P_{1}}\right)=1$ for monic irreducible divisor $P_{1}$ of $D_{F}$ and $D_{F}$ has two monic irreducible divisors $P_{2}, P_{3}$ which are different from $P_{1}$ and $P_{i} \nmid D_{1} D_{2}$ $(i=2,3)$, then $F$ has an infinite Hilbert 2-class field tower.

Proof. Let $K=k\left(\sqrt{D_{1}}, \sqrt{D_{2}}\right)$ and $E=K F$. As in the proof of Corollary 2.3, we can show that $E$ is contained in $F_{1}^{(2)}$. Since $\left(\frac{D_{1}}{P_{1}}\right)=\left(\frac{D_{2}}{P_{1}}\right)=1, P_{1}$ splits completely in $K$. Each $P_{i}(i=2,3)$ splits in at least one of $k\left(\sqrt{D_{1}}\right), k\left(\sqrt{D_{2}}\right)$ and $k\left(\sqrt{D_{1} D_{2}}\right)$. Hence we have $\gamma_{E / K} \geq 8$ and $\left|S_{\infty}(K)\right|=\rho_{E / K}=4$. Then by applying Proposition 2.1, $E$ has an infinite Hilbert 2-class field tower. Since $E$ is contained in $F_{1}^{(2)}, F$ also has an infinite Hilbert 2-class field tower.

\subsection{Rédei-matrix and 4-rank of class group}

Let $F$ be an imaginary quadratic function field over $k$ with $D_{F}=\gamma^{a} P_{1} \cdots P_{t}$. Let $d_{i} \in \mathbb{F}_{2}$ be defined by $d_{i} \equiv \operatorname{deg} P_{i} \bmod 2$ for $1 \leq i \leq t$. We will separate two cases:

- $(\mathrm{CASE}$ A) $q \equiv 1 \bmod 4$ with $a=0$ or $q \equiv 3 \bmod 4$ with $a=1$,

- $(\mathrm{CASE} \mathrm{B}) q \equiv 1 \bmod 4$ with $a=1$ or $q \equiv 3 \bmod 4$ with $a=0$.

We associate a $t \times t$ matrix $R_{F}=\left(e_{i j}\right)_{1 \leq i, j \leq t}$ over $\mathbb{F}_{2}$ to $F$, where $e_{i j} \in \mathbb{F}_{2}$ is defined by $(-1)^{e_{i j}}=\left(\frac{P_{i}}{P_{j}}\right)$ for $1 \leq i \neq j \leq t$ and the diagonal entries $e_{i i} \in \mathbb{F}_{2}$ are defined to satisfy the relation

$$
\sum_{j=1}^{t} e_{i j}= \begin{cases}0 & (\text { CASE A }) \\ d_{i} & (\text { CASE B })\end{cases}
$$

Let $s_{F}$ be the number of monic irreducible divisors $P_{i}$ of $D_{F}$ of odd degree. Since $\operatorname{deg} D_{F}$ is odd, $s_{F}$ is an odd integer. We will assume that $\operatorname{deg} P_{i}$ is odd for $1 \leq i \leq s_{F}$. If $q \equiv 3 \bmod 4$ and $1 \leq j \leq s_{F}$, we have

$$
\sum_{i=1}^{t} e_{i j}=e_{j j}+\sum_{1 \leq i \leq s_{F}, i \neq j}\left(e_{j i}+1\right)+\sum_{i=s_{F}+1}^{t} e_{j i}=\sum_{i=1}^{t} e_{j i}
$$


since $s_{F}$ is odd. Otherwise, since $e_{i j}=e_{j i}$, we trivially have the same equality. Hence, the diagonal entries $e_{i i}$ of $R_{F}$ also satisfy the relation

$$
\sum_{i=1}^{t} e_{i j}= \begin{cases}0 & (\mathrm{CASE} \mathrm{A}) \\ d_{j} & (\mathrm{CASE} \mathrm{B})\end{cases}
$$

Proposition 2.5. For an imaginary quadratic function field $F$ over $k$, we have

$$
r_{4}\left(\mathcal{C} l_{F}\right)= \begin{cases}t-1-\operatorname{rank} R_{F} & (\text { CASE A }), \\ t-\operatorname{rank} R_{F} & (\text { CASE B }) .\end{cases}
$$

Proof. Let $M_{F}$ be the $(t+1) \times(t+1)$ matrix over $\mathbb{F}_{2}$ given by

$$
M_{F}=\left(\begin{array}{cccc}
e_{11} & \cdots & e_{1 t} & (a+\epsilon) d_{1} \\
\vdots & & \vdots & \vdots \\
e_{t 1} & \cdots & e_{t t} & (a+\epsilon) d_{t} \\
d_{1} & \cdots & d_{t} & 1
\end{array}\right)
$$

where $\epsilon=0$ if $q \equiv 1 \bmod 4, \epsilon=1$ if $q \equiv 3 \bmod 4$ and the entries $e_{i i} \in \mathbb{F}_{2}$ are defined to satisfy $(a+\epsilon) d_{i}+\sum_{j=1}^{t} e_{i j}=0$. Then $r_{4}\left(\mathcal{C} l_{F}\right)$ satisfies the following equality ([1, Corollary 3.8]):

$$
r_{4}\left(\mathcal{C} l_{F}\right)=t-\operatorname{rank} M_{F} .
$$

In (CASE A), we have $a+\epsilon=0$ in $\mathbb{F}_{2}$. Hence we can see that $\operatorname{rank} M_{F}=$ $\operatorname{rank} R_{F}+1$ and the entries $e_{i i}$ satisfy $\sum_{j=1}^{t} e_{i j}=0$. By (2.2), we have $r_{4}\left(\mathcal{C} l_{F}\right)=t-1-\operatorname{rank} R_{F}$.

Now, we consider (CASE B). Then $a+\epsilon=1$ in $\mathbb{F}_{2}$, so

$$
M_{F}=\left(\begin{array}{cccc}
e_{11} & \cdots & e_{1 t} & d_{1} \\
\vdots & & \vdots & \vdots \\
e_{t 1} & \cdots & e_{t t} & d_{t} \\
d_{1} & \cdots & d_{t} & 1
\end{array}\right)
$$

and the entries $e_{i i}$ satisfy $d_{i}+\sum_{j=1}^{t} e_{i j}=0$. By adding first $t$ columns to the last column on $M_{F}$, we can see that $\operatorname{rank} M_{F}=\operatorname{rank} M_{F}^{\prime}$, where

$$
M_{F}^{\prime}=\left(\begin{array}{ccc}
e_{11} & \cdots & e_{1 t} \\
\vdots & & \vdots \\
e_{t 1} & \cdots & e_{t t} \\
d_{1} & \cdots & d_{t}
\end{array}\right)
$$

By adding first $t$ rows to the last row on $M_{F}^{\prime}$, we can see that $\operatorname{rank} M_{F}^{\prime}=$ $\operatorname{rank} R_{F}$, so $r_{4}\left(\mathcal{C} l_{F}\right)=t-\operatorname{rank} R_{F}$ by $(2.2)$. 


\subsection{Some asymptotic results}

Let $\mathcal{P}$ be the set of all monic irreducible polynomials in $\mathbb{A}$. For positive integers $n$ and $t$, we write $\mathcal{P}(n)$ for the subset of $\mathcal{P}$ consisting of $P \in \mathcal{P}$ with $\operatorname{deg} P=n, \mathcal{P}(n, t)$ for the set of monic square free polynomials $N \in \mathbb{A}$ with $\operatorname{deg} N=n$ and $\omega(N)=t$ and $\mathcal{P}^{\prime}(n, t)$ for the subset of $\mathcal{P}(n, t)$ consisting of $N=P_{1} \cdots P_{t} \in \mathcal{P}(n, t)$ such that $\operatorname{deg}\left(P_{i}\right) \neq \operatorname{deg}\left(P_{j}\right)$ for $i \neq j$. As $n \rightarrow \infty$, we have

$$
\begin{aligned}
& |\mathcal{P}(n, t)|=\frac{q^{n}(\log n)^{t-1}}{(t-1) ! n}+O\left(\frac{q^{n}(\log n)^{t-2}}{n}\right), \\
& \left|\mathcal{P}(n, t) \backslash \mathcal{P}^{\prime}(n, t)\right|=o\left(\frac{q^{n}(\log n)^{t-1}}{n}\right) .
\end{aligned}
$$

The following two lemmas are due to Wittmann ([10, Lemmas 3.3 and 3.5]).

Lemma 2.6. For $P_{1}, \ldots, P_{u} \in \mathcal{P}$ and $\varepsilon_{1}, \ldots, \varepsilon_{u} \in\{ \pm 1\}$, as $n \rightarrow \infty$, we have

$$
\sum_{\substack{P \in \mathcal{P}(n) \\ 1 \leq i \leq u:\left(P_{i} / P\right)=\varepsilon_{i}}} 1=2^{-u} \frac{q^{n}}{n}+O\left(\frac{q^{n / 2}}{n}\right) .
$$

Lemma 2.7. Let $n$ be a positive integer and $d_{1}, \ldots, d_{t} \in\{0,1\}$ satisfying $\sum_{i=1}^{t} d_{i} \equiv n \bmod 2$. Then, as $n \rightarrow \infty$, we have

$$
\sum_{\substack{0<n_{1}<\cdots<n_{t} \\ n_{1} \equiv d_{1}(2), \ldots, n_{t} \equiv d_{t}(2) \\ n_{1}+\cdots+n_{t}=n}} \frac{1}{n_{1} \cdots n_{t}}=2^{-(t-1)} \frac{(\log n)^{t-1}}{(t-1) ! n}+O\left(\frac{(\log n)^{t-2}}{n}\right) .
$$

For $d_{1}, \ldots, d_{t} \in\{0,1\}$ and $\varepsilon_{i j} \in\{ \pm 1\}$ for $1 \leq i<j \leq t$, let

$$
J_{n}\left(\left\{d_{i}\right\} ;\left\{\varepsilon_{i j}\right\}\right)=\sum_{\substack{0<n_{1}<\cdots<n_{t} \\ n_{1} \equiv d_{1}(2), \ldots, n_{t} \equiv d_{t}(2) \\ n_{1}+\cdots+n_{t}=n^{\prime}}} \sum_{P_{1} \in \mathcal{P}\left(n_{1}\right)} \sum_{\substack{P_{2} \in \mathcal{P}\left(n_{2}\right) \\\left(P_{1} / P_{2}\right)=\varepsilon_{12}}} \ldots \sum_{\substack{P_{t} \in \mathcal{P}\left(n_{t}\right) \\ \forall i<t:\left(P_{i} / P_{t}\right)=\varepsilon_{i t}}} 1 .
$$

Lemma 2.8. As $n \rightarrow \infty$, we have

$$
J_{n}\left(\left\{d_{i}\right\} ;\left\{\varepsilon_{i j}\right\}\right)=2^{1-\frac{t^{2}+t}{2}} \frac{q^{n}(\log n)^{t-1}}{(t-1) ! n}+O\left(\frac{q^{n}(\log n)^{t-2}}{n}\right) .
$$

Proof. Let $J=J_{n}\left(\left\{d_{i}\right\} ;\left\{\varepsilon_{i j}\right\}\right)$. As $n \rightarrow \infty$, using Lemmas 2.6 and 2.7, we get

$$
\begin{aligned}
J & =\sum_{\substack{0<n_{1}<\cdots<n_{t} \\
n_{1} \equiv d_{1}(2), \ldots, n_{t} \equiv d_{t}(2) \\
n_{1}+\cdots+n_{t}=n}} \prod_{i=1}^{t}\left(2^{-(i-1)} \frac{q^{n_{i}}}{n_{i}}+O\left(\frac{q^{n_{i} / 2}}{n_{i}}\right)\right) \\
& =2^{-\frac{\left(t^{2}-t\right)}{2}} q^{n} \sum_{\substack{0<n_{1}<\cdots<n_{t} \\
n_{1} \equiv d_{1}(2), \ldots, n_{t} \equiv d_{t}(2) \\
n_{1}+\cdots+n_{t}=n}} \frac{1}{n_{1} \cdots n_{t}}+O\left(q^{n} \sum_{i=1}^{t} \sum_{\substack{0<n_{1}<\cdots<n_{t} \\
n_{1}+\cdots+n_{t}=n}} \frac{q^{-n_{i} / 2}}{n_{1} \cdots n_{t}}\right) \\
& =2^{1-\frac{t^{2}+t}{2}} \frac{q^{n}(\log n)^{t-1}}{(t-1) ! n}+O\left(\frac{q^{n}(\log n)^{t-2}}{n}\right) .
\end{aligned}
$$


For $N=P_{1} \cdots P_{t}, N^{\prime}=P_{1}^{\prime} \cdots P_{t}^{\prime} \in \mathcal{P}^{\prime}(n, t)$, we say that $N$ and $N^{\prime}$ are equivalent if $\operatorname{deg}\left(P_{i}\right) \equiv \operatorname{deg}\left(P_{i}^{\prime}\right) \bmod 2$ for $1 \leq i \leq t$ and $\left(\frac{P_{i}}{P_{j}}\right)=\left(\frac{P_{i}^{\prime}}{P_{j}^{\prime}}\right)$ for $1 \leq i<j \leq t$. Write $\mathcal{N}(N)$ for the set of polynomials in $\mathcal{P}^{\prime}(n, t)$ which are equivalent to $N$.

Proposition 2.9. For any $N \in \mathcal{P}^{\prime}(n, t)$, as $n \rightarrow \infty$, we have

$$
|\mathcal{N}(N)|=2^{1-\frac{\left(t^{2}+t\right)}{2}} \frac{q^{n}(\log n)^{t-1}}{(t-1) ! n}+O\left(\frac{q^{n}(\log n)^{t-2}}{n}\right) .
$$

Proof. Let $N=P_{1} \cdots P_{t} \in \mathcal{P}^{\prime}(n, t)$. Then we have $|\mathcal{N}(N)|=J_{n}\left(\left\{d_{i}\right\} ;\left\{\varepsilon_{i j}\right\}\right)$, where $d_{1}, \ldots, d_{t} \in\{0,1\}$ satisfying $\operatorname{deg} P_{i} \equiv d_{i} \bmod 2$ for $1 \leq i \leq t$ and $\varepsilon_{i j}=$ $\left(\frac{P_{i}}{P_{j}}\right)$ for $1 \leq i<j \leq t$. Now, the result follows immediately from Lemma 2.8 .

\section{Proof of Theorem 1.2}

Let $F$ be an imaginary quadratic function field with $D_{F}=\gamma^{a} P_{1} \cdots P_{t}$. By Theorem 1.1, the Hilbert 2-class field tower of $F$ is infinite if $r_{2}\left(\mathcal{C} l_{F}\right) \geq 5$. Hence, it remains to consider the cases $\left(r_{2}\left(\mathcal{C} l_{F}\right), r_{4}\left(\mathcal{C} l_{F}\right)\right)=(3,3),(4,3)$ or $(4,4)$. Recall that $s_{F}$ denotes the number of monic irreducible divisors $P_{i}$ of $D_{F}$ of odd degree. Since $\operatorname{deg} D_{F}$ is odd, $s_{F}$ is an odd integer. We will assume that $\operatorname{deg} P_{i}$ is odd for $1 \leq i \leq s_{F}$. Write $\vec{R}_{i}$ for the $i$-th row vector of $R_{F}$ and $\overrightarrow{0}$ for the zero row vector. In (CASE B), we always have $\vec{R}_{i} \neq \overrightarrow{0}$ for $1 \leq i \leq s_{F}$ since $e_{i 1}+\cdots+e_{i t}=1$. In the following proof, we will consider the cases that $\operatorname{rank} R_{F}=0,1$ or 2 .

- If rank $R_{F}=0$, then $R_{F}=O$, so we have $\left(\frac{P_{i}}{P_{j}}\right)=1$ for all $1 \leq i \neq j \leq t$.

- If rank $R_{F}=1$, then any nonzero row of $R_{F}$ forms a basis for the row space of $R_{F}$. Especially, $\left\{\vec{R}_{1}\right\}$ is always a basis for the row space of $R_{F}$ in (CASE B).

- If rank $R_{F}=2$, then any two distinct nonzero rows of $R_{F}$ forms a basis for the row space of $R_{F}$.

\subsection{Case $r_{2}\left(\mathcal{C} l_{F}\right)=r_{4}\left(\mathcal{C} l_{F}\right)=3$ with $D_{F}=\gamma^{a} P_{1} P_{2} P_{3} P_{4}$}

We first consider (CASE A). By Proposition 2.5, we have $\operatorname{rank} R_{F}=0$, i.e., $R_{F}=O$. If $s_{F}=1$, we have $\left(\frac{P_{3}}{P_{i}}\right)=\left(\frac{P_{4}}{P_{i}}\right)=1$ for $i \in\{1,2\}$, so $F$ has an infinite Hilbert 2-class field tower by Corollary 2.3. If $s_{F}=3$, we have $q \equiv 1 \bmod 4$ since $\left(\frac{P_{1}}{P_{2}}\right)=\left(\frac{P_{2}}{P_{1}}\right)=1$. In this case, we suppose that $\operatorname{deg} P_{4}$ is divisible by 4 and $\left(\frac{P_{4}}{P_{1}}\right)_{4}=1$. Then $\infty$ and $P_{1}$ splits completely in $K=k\left(\sqrt[4]{P_{4}}\right)$. Since $\left(\frac{P_{4}}{P_{2}}\right)=\left(\frac{P_{4}}{P_{3}}\right)=1, P_{2}$ and $P_{3}$ split in $k\left(\sqrt{P_{4}}\right)$. Put $E=K F$. Then $E$ is contained in $F_{1}^{(2)}$. By applying Proposition 2.1 on $E / K$ with $\gamma_{E / K} \geq 8$ and $\left|S_{\infty}(K)\right|=\rho_{E / K}=4$, we see that $E$ has an infinite Hilbert 2-class field tower. Since $E$ is contained in $F_{1}^{(2)}, F$ also has an infinite Hilbert 2-class field tower. 
Now, we consider (CASE B). By Proposition 2.5, we have rank $R_{F}=1$. Since $\vec{R}_{1}+\vec{R}_{2}+\vec{R}_{3}+\vec{R}_{4}=\vec{e}$, where

$$
\vec{e}=\left\{\begin{array}{lll}
\left(\begin{array}{llll}
1 & 0 & 0 & 0
\end{array}\right) & \text { if } s_{F}=1 \\
\left(\begin{array}{llll}
1 & 1 & 1 & 0
\end{array}\right) & \text { if } s_{F}=3
\end{array}\right.
$$

we have $\vec{R}_{i} \in\{\overrightarrow{0}, \vec{e}\}$ for $1 \leq i \leq 4$. If $s_{F}=1$, we have $\vec{R}_{1}=\vec{e}$ and $\vec{R}_{2}=$ $\vec{R}_{3}=\vec{R}_{4}=\overrightarrow{0}$. Then we have $\left(\frac{P_{3}}{P_{i}}\right)=\left(\frac{P_{4}}{P_{i}}\right)=1$ for $i \in\{1,2\}$, so $F$ has an infinite Hilbert 2-class field tower by Corollary 2.3. If $s_{F}=3$, we have $\vec{R}_{1}=\vec{R}_{2}=\vec{R}_{3}=\vec{e}$ and $\vec{R}_{4}=0$. We also have $q \equiv 1 \bmod 4$ since $\left(\frac{P_{1}}{P_{2}}\right)=\left(\frac{P_{2}}{P_{1}}\right)$. In this case, as in above (CASE A), we can show that $F$ also has an infinite Hilbert 2-class field tower.

\subsection{Case $\left.r_{2}\left(\mathcal{C} l_{F}\right)=r_{4}\left(\mathcal{C} l_{F}\right)\right)=4$ with $D_{F}=\gamma^{a} P_{1} P_{2} P_{3} P_{4} P_{5}$}

First consider (CASE A). By Proposition 2.5, we have $\operatorname{rank} R_{F}=0$, i.e., $R_{F}=O$. If $s_{F} \leq 3$, then $\left(\frac{P_{5}}{P_{i}}\right)=1$ for $1 \leq i \leq 4$, so $F$ has an infinite Hilbert 2-class field tower by Corollary 2.2. If $s_{F}=5$, for $D_{1}=P_{1} P_{2}$ and $D_{2}=P_{1} P_{3}$, we have $\left(\frac{D_{1}}{P_{i}}\right)=\left(\frac{D_{2}}{P_{i}}\right)=1$ for $i \in\{4,5\}$, so $F$ has an infinite Hilbert 2-class field tower by Corollary 2.3.

Consider (CASE B). By Proposition 2.5, we have rank $R_{F}=1$. Since $\left\{\vec{R}_{1}\right\}$ is a basis for the row space of $R_{F}$ and $\vec{R}_{1}+\vec{R}_{2}+\vec{R}_{3}+\vec{R}_{4}+\vec{R}_{5}=\vec{f}$, where

$$
\vec{f}=\left\{\begin{array}{lll}
\left(\begin{array}{lllll}
1 & 0 & 0 & 0 & 0
\end{array}\right) & \text { if } s_{F}=1 \\
\left(\begin{array}{lllll}
1 & 1 & 1 & 0 & 0
\end{array}\right) & \text { if } s_{F}=3 \\
\left(\begin{array}{lllll}
1 & 1 & 1 & 1 & 1
\end{array}\right) & \text { if } s_{F}=5
\end{array}\right.
$$

we have $\vec{R}_{1}=\vec{f}$. If $s_{F} \leq 3$, we have $\vec{R}_{5}=\overrightarrow{0}$ since $e_{51}=e_{15}=0$. Then $\left(\frac{P_{5}}{P_{i}}\right)=1$ for $1 \leq i \leq 4$, so so $F$ has an infinite Hilbert 2-class field tower by Corollary 2.2. If $s_{F}=5$, we have $\vec{R}_{i}=\vec{f}$ for $1 \leq i \leq 5$. For $D_{1}=P_{1} P_{2}$ and $D_{2}=P_{1} P_{3}$, we have $\left(\frac{D_{1}}{P_{i}}\right)=\left(\frac{D_{2}}{P_{i}}\right)=1$ for $i \in\{4,5\}$, so $F$ has an infinite Hilbert 2-class field tower by Corollary 2.3 .

\subsection{Case $r_{2}\left(\mathcal{C} l_{F}\right)=4$ and $r_{4}\left(\mathcal{C} l_{F}\right)=3$ with $D_{F}=\gamma^{a} P_{1} P_{2} P_{3} P_{4} P_{5}$}

First consider (CASE A). By Proposition 2.5, we have rank $R_{F}=1$. Assume $s_{F}=1$. If $\vec{R}_{i}=\overrightarrow{0}$ for some $2 \leq i \leq 5$, say $\vec{R}_{5}=\overrightarrow{0}$, then $\left(\frac{P_{5}}{P_{i}}\right)=1$ for $1 \leq i \leq 4$, so $F$ has an infinite Hilbert 2-class field tower by Corollary 2.2. Otherwise, we have $\vec{R}_{2}=\vec{R}_{3}=\vec{R}_{4}=\vec{R}_{5} \neq \overrightarrow{0}$. For $D_{1}=P_{3} P_{4}$ and $D_{2}=P_{3} P_{5}$, we have $\left(\frac{D_{1}}{P_{i}}\right)=\left(\frac{D_{2}}{P_{i}}\right)=1$ for $i \in\{1,2\}$, so $F$ has an infinite Hilbert 2-class field tower by Corollary 2.3 .

Assume $s_{F}=3$. If $\vec{R}_{4}=\overrightarrow{0}$ or $\vec{R}_{5}=\overrightarrow{0}$, say $\vec{R}_{5}=\overrightarrow{0}$, then $\left(\frac{P_{5}}{P_{i}}\right)=1$ for $1 \leq i \leq 4$, so $F$ has an infinite Hilbert 2-class field tower by Corollary 2.2. We may assume $\vec{R}_{4}=\vec{R}_{5} \neq \overrightarrow{0}$. If $\vec{R}_{1}=\vec{R}_{2}=\vec{R}_{3}$, for $D_{1}=P_{1} P_{2}$ and $D_{2}=P_{1} P_{3}$, 
we have $\left(\frac{D_{1}}{P_{i}}\right)=\left(\frac{D_{2}}{P_{i}}\right)=1$ for $i \in\{4,5\}$, so $F$ has an infinite Hilbert 2class field tower by Corollary 2.3. We may assume $\vec{R}_{1} \neq \vec{R}_{2}=\vec{R}_{3}$. Since $\vec{R}_{1}+\vec{R}_{2}+\vec{R}_{3}+\vec{R}_{4}+\vec{R}_{5}=\overrightarrow{0}$, we have $\vec{R}_{1}=\overrightarrow{0}$ and $\vec{R}_{2}=\vec{R}_{3}=\vec{R}_{4}=\vec{R}_{5} \neq \overrightarrow{0}$. Then we have $q \equiv 1 \bmod 4$ and $a=0$. Since $D_{F} / P_{1}$ is monic of even degree and $\left(\frac{P_{1}}{P_{i}}\right)=1$ for $2 \leq i \leq 5, F$ has an infinite Hilbert 2-class field tower by Corollary 2.2.

If $s_{F}=5$, then at least three rows of $R_{F}$ are equal, say $\vec{R}_{3}=\vec{R}_{4}=\vec{R}_{5}$. For $D_{1}=P_{3} P_{4}$ and $D_{2}=P_{3} P_{5}$, we have $\left(\frac{D_{1}}{P_{i}}\right)=\left(\frac{D_{2}}{P_{i}}\right)=1$ for $i \in\{1,2\}$, so $F$ has an infinite Hilbert 2-class field tower by Corollary 2.3.

Now we consider (CASE B). In this case we have rank $R_{F}=2$ by Proposition 2.5. Assume $s_{F}=1$. If $\vec{R}_{i}=\overrightarrow{0}$ for some $2 \leq i \leq 5$, say $\vec{R}_{5}=\overrightarrow{0}$, then $\left(\frac{P_{5}}{P_{i}}\right)=1$ for $1 \leq i \leq 4$, so $F$ has an infinite Hilbert 2-class field tower by Corollary 2.2. We may assume $\vec{R}_{i} \neq \overrightarrow{0}$ for $2 \leq i \leq 5$. It only need to consider the case that either $\left\{\vec{R}_{1}, \vec{f}\right\}$ or $\left\{\vec{R}_{5}, \vec{f}\right\}$ is a basis of the row space of $R_{F}$. In any case, since $\vec{R}_{i} \neq \overrightarrow{0}$ and $e_{i 1}+e_{i 2}+e_{i 3}+e_{i 4}+e_{i 5}=0$ for $2 \leq i \leq 5$, we have $\vec{R}_{2}=\vec{R}_{3}=\vec{R}_{4}=\vec{R}_{5}$. For $D_{1}=P_{3} P_{4}$ and $D_{2}=P_{3} P_{5}$, we have $\left(\frac{D_{1}}{P_{i}}\right)=\left(\frac{D_{2}}{P_{i}}\right)=1$ for $i \in\{1,2\}$, so $F$ has an infinite Hilbert 2-class field tower by Corollary 2.3.

Assume $s_{F}=3$. If $\vec{R}_{4}=\overrightarrow{0}$ or $\vec{R}_{5}=\overrightarrow{0}$, say $\vec{R}_{5}=\overrightarrow{0}$, then $\left(\frac{P_{5}}{P_{i}}\right)=1$ for $1 \leq i \leq 4$, so $F$ has an infinite Hilbert 2-class field tower by Corollary 2.2. We may assume $\vec{R}_{i} \neq \overrightarrow{0}$ for $i \in\{4,5\}$. Then $\left\{\vec{R}_{1}, \vec{R}_{4}\right\}$ forms a basis of the row space of $R_{F}$, so we have $\vec{R}_{5}=\vec{R}_{4}$ and $\vec{R}_{i} \in\left\{\vec{R}_{1}, \vec{R}_{1}+\vec{R}_{4}\right\}$ for $i \in\{2,3\}$. If $\vec{R}_{1}=\vec{R}_{2}=\vec{R}_{3}$, then, for $D_{1}=P_{1} P_{2}$ and $D_{2}=P_{1} P_{3}$, we have $\left(\frac{D_{1}}{P_{i}}\right)=\left(\frac{D_{2}}{P_{i}}\right)=1$ for $i \in\{4,5\}$, so $F$ has an infinite Hilbert 2-class field tower by Corollary 2.3. By changing the role of $P_{1}$ and $P_{3}$ if it is necessary, we are reduced to the case $\vec{R}_{1}=\vec{R}_{2} \neq \vec{R}_{3}$. Since $\vec{R}_{1}+\vec{R}_{2}+\vec{R}_{3}+\vec{R}_{4}+\vec{R}_{5}=\vec{f}$, we have $\vec{R}_{3}=\vec{f}$ and $\vec{R}_{1}=\vec{R}_{2}=\vec{f}+\vec{R}_{4}$. By using the polynomial quadratic reciprocity law, we can see that $q \equiv 1 \bmod 4$ and $\vec{R}_{4}=\vec{R}_{5}=\left(\begin{array}{lllll}1 & 1 & 0 & 1 & 1\end{array}\right)$. Then $\left(\frac{P_{4}}{P_{3}}\right)=\left(\frac{P_{5}}{P_{3}}\right)=1$, so $F$ has an infinite Hilbert 2-class field tower by Corollary 2.4.

If $s_{F}=5$, since $\vec{R}_{i} \neq \overrightarrow{0}$ and $e_{i 1}+e_{i 2}+e_{i 3}+e_{i 4}+e_{i 5}=1$ for $1 \leq i \leq 5$, at least three of rows of $R_{F}$ are equal, say $\vec{R}_{3}=\vec{R}_{4}=\vec{R}_{5}$. For $D_{1}=P_{3} P_{4}$ and $D_{2}=P_{3} P_{5}$, we have $\left(\frac{D_{1}}{P_{i}}\right)=\left(\frac{D_{2}}{P_{i}}\right)=1$ for $i \in\{1,2\}$, so $F$ has an infinite Hilbert 2-class field tower by Corollary 2.3.

\section{Proof of Theorem 1.3}

Let $r, s$ be integers with $0 \leq s \leq r$ and $n$ be a positive odd integer. We have

$$
X_{r ; n}=\left\{k\left(\sqrt{\gamma^{a} N}\right): a \in\{0,1\} \text { and } N \in \mathcal{P}(n, r+1)\right\} .
$$

Let $\bar{X}_{r ; n}$ be the subset of $X_{r ; n}$ consisting of $k\left(\sqrt{\gamma^{a} N}\right) \in X_{r ; n}$ with $N \in \mathcal{P}^{\prime}(n, r+$ 1) and $\bar{X}_{r, s ; n}^{*}=\bar{X}_{r ; n} \cap X_{r, s ; n}^{*}$. Then, by (2.4), we have

$$
\delta_{r, s}^{*}=\liminf _{\substack{n \rightarrow \infty \\ n: \text { odd }}} \frac{\left|\bar{X}_{r, s ; n}^{*}\right|}{\left|\bar{X}_{r ; n}\right|} .
$$


By (2.3) and (2.4), we have

$$
\left|\bar{X}_{r ; n}\right|=\frac{2 q^{n}(\log n)^{r}}{r ! n}+O\left(\frac{q^{n}(\log n)^{r-1}}{n}\right) \quad \text { as } n \rightarrow \infty .
$$

For any $N \in \mathcal{P}^{\prime}(n, r+1)$, let

$$
\mathcal{S}_{a}(N)=\left\{k\left(\sqrt{\gamma^{a} N^{\prime}}\right): N^{\prime} \in \mathcal{N}(N)\right\} \quad(a=0,1) .
$$

Then $\mathcal{S}_{0}(N) \cup \mathcal{S}_{1}(N)$ is a subset of $\bar{X}_{r ; n}$ and by Proposition 2.9, we have

$$
\left|\mathcal{S}_{0}(N)\right|=\left|\mathcal{S}_{1}(N)\right|=2^{-\frac{r(r+3)}{2}} \frac{q^{n}(\log n)^{r}}{r ! n}+O\left(\frac{q^{n}(\log n)^{r-1}}{n}\right) \quad \text { as } n \rightarrow \infty .
$$

By (4.2) and (4.3), we have

$$
\lim _{\substack{n \rightarrow \infty \\ n: \text { odd }}} \frac{\left|\mathcal{S}_{0}(N)\right|}{\left|\bar{X}_{r ; n}\right|}=\lim _{\substack{n \rightarrow \infty \\ n: \text { odd }}} \frac{\left|\mathcal{S}_{1}(N)\right|}{\left|\bar{X}_{r ; n}\right|}=2^{-\frac{(r+1)(r+2)}{2}} .
$$

\section{1. $\delta_{3, s}^{*}$}

Consider $F_{a}=k\left(\sqrt{\gamma^{a} N}\right)(a=0,1)$, where $N=P_{1} P_{2} P_{3} P_{4} \in \mathcal{P}^{\prime}(n, 4)$ such that $2 \mid \operatorname{deg} P_{i}$ for $1 \leq i \leq 3,2 \nmid \operatorname{deg} P_{4}$ and $\left(\frac{P_{j}}{P_{i}}\right)=1$ for $i=1,2$ and $j=3,4$. Then $r_{2}\left(\mathcal{C} l_{F_{a}}\right)=3$ and $F_{a}$ has an infinite Hilbert 2-class field tower by Lemma 2.3. Moreover, every fields in $\mathcal{S}_{a}(N)$ also have infinite Hilbert 2-class field towers.

- $\operatorname{Case}\left(\frac{P_{2}}{P_{1}}\right)=\left(\frac{P_{4}}{P_{3}}\right)=-1$. We have

$$
R_{F_{0}}=\left(\begin{array}{cccc}
1 & 1 & 0 & 0 \\
1 & 1 & 0 & 0 \\
0 & 0 & 1 & 1 \\
0 & 0 & 1 & 0
\end{array}\right), \quad R_{F_{1}}=\left(\begin{array}{cccc}
1 & 1 & 0 & 0 \\
1 & 1 & 0 & 0 \\
0 & 0 & 1 & 1 \\
0 & 0 & 1 & 1
\end{array}\right)
$$

with $\operatorname{rank} R_{F_{0}}=3$ and $\operatorname{rank} R_{F_{1}}=2$, so $r_{4}\left(\mathcal{C} l_{F_{0}}\right)=r_{4}\left(\mathcal{C} l_{F_{1}}\right)=1$. Hence $F_{0}, F_{1} \in \bar{X}_{3,1 ; n}^{*}$ and $\mathcal{S}_{0}(N) \cup \mathcal{S}_{1}(N) \subset \bar{X}_{3,1 ; n}^{*}$, so we have

$$
\delta_{3,1}^{*} \geq \lim _{\substack{n \rightarrow \infty \\ n: \text { odd }}} \frac{\left|\mathcal{S}_{0}(N)\right|}{\left|\bar{X}_{3 ; n}\right|}+\lim _{\substack{n \rightarrow \infty \\ n: \text { odd }}} \frac{\left|\mathcal{S}_{1}(N)\right|}{\left|\bar{X}_{3 ; n}\right|}=2^{-9} .
$$

- $\operatorname{Case}\left(\frac{P_{2}}{P_{1}}\right)=-1$ and $\left(\frac{P_{4}}{P_{3}}\right)=1$. We have

$$
R_{F_{0}}=\left(\begin{array}{cccc}
1 & 1 & 0 & 0 \\
1 & 1 & 0 & 0 \\
0 & 0 & 0 & 0 \\
0 & 0 & 0 & 1
\end{array}\right), \quad R_{F_{1}}=\left(\begin{array}{cccc}
1 & 1 & 0 & 0 \\
1 & 1 & 0 & 0 \\
0 & 0 & 0 & 0 \\
0 & 0 & 0 & 0
\end{array}\right)
$$

with $\operatorname{rank} R_{F_{0}}=2$ and $\operatorname{rank} R_{F_{0}}=1$, so $r_{4}\left(\mathcal{C} l_{F_{0}}\right)=r_{4}\left(\mathcal{C} l_{F_{1}}\right)=2$. Hence $F_{0}, F_{1} \in \bar{X}_{3,2 ; n}^{*}$ and $\mathcal{S}_{0}(N) \cup \mathcal{S}_{1}(N) \subset \bar{X}_{3,2 ; n}^{*}$, so we have

$$
\delta_{3,2}^{*} \geq \lim _{\substack{n \rightarrow \infty \\ n: \text { odd }}} \frac{\left|\mathcal{S}_{0}(N)\right|}{\left|\bar{X}_{3 ; n}\right|}+\lim _{\substack{n \rightarrow \infty \\ n: \text { odd }}} \frac{\left|\mathcal{S}_{1}(N)\right|}{\left|\bar{X}_{3 ; n}\right|}=2^{-9}
$$


- $\operatorname{Case}\left(\frac{P_{2}}{P_{1}}\right)=\left(\frac{P_{4}}{P_{3}}\right)=1$. We have

$$
R_{F_{0}}=\left(\begin{array}{cccc}
0 & 0 & 0 & 0 \\
0 & 0 & 0 & 0 \\
0 & 0 & 0 & 0 \\
0 & 0 & 0 & 1
\end{array}\right), \quad R_{F_{1}}=\left(\begin{array}{cccc}
0 & 0 & 0 & 0 \\
0 & 0 & 0 & 0 \\
0 & 0 & 0 & 0 \\
0 & 0 & 0 & 0
\end{array}\right)
$$

with $\operatorname{rank} R_{F_{0}}=1$ and $\operatorname{rank} R_{F_{1}}=0$, so $r_{4}\left(\mathcal{C} l_{F_{0}}\right)=r_{4}\left(\mathcal{C} l_{F_{1}}\right)=3$. Hence $F_{0}, F_{1} \in \bar{X}_{3,3 ; n}^{*}$ and $\mathcal{S}_{0}(N) \cup \mathcal{S}_{1}(N) \subset \bar{X}_{3,3 ; n}^{*}$, so we have

$$
\delta_{3,3}^{*} \geq \lim _{\substack{n \rightarrow \infty \\ n: \text { odd }}} \frac{\left|\mathcal{S}_{0}(N)\right|}{\left|\bar{X}_{3 ; n}\right|}+\lim _{\substack{n \rightarrow \infty \\ n: \text { odd }}} \frac{\left|\mathcal{S}_{1}(N)\right|}{\left|\bar{X}_{3 ; n}\right|}=2^{-9}
$$

\section{2. $\delta_{4, s}^{*}$}

Now consider $F_{a}=k\left(\sqrt{\gamma^{a} N}\right)(a=0,1)$, where $N=P_{1} P_{2} P_{3} P_{4} P_{5} \in \mathcal{P}^{\prime}(n, 5)$ such that $2 \mid \operatorname{deg} P_{i}$ for $1 \leq i \leq 4,2 \nmid \operatorname{deg} P_{5}$ and $\left(\frac{P_{j}}{P_{i}}\right)=1$ for $1 \leq i<j \leq 4$. Then $r_{2}\left(\mathcal{C} l_{F_{a}}\right)=4$ and $F_{a}$ has an infinite Hilbert 2-class field tower by Lemma 2.3, so every field in the set $\mathcal{S}_{a}(N)$ also has an infinite Hilbert 2-class field tower.

- Case $\left(\frac{P_{5}}{P_{i}}\right)=-1$ for $1 \leq i \leq 4$. We have

$$
R_{F_{0}}=\left(\begin{array}{ccccc}
1 & 0 & 0 & 0 & 1 \\
0 & 1 & 0 & 0 & 1 \\
0 & 0 & 1 & 0 & 1 \\
0 & 0 & 0 & 1 & 1 \\
1 & 1 & 1 & 1 & 1
\end{array}\right), \quad R_{F_{0}}=\left(\begin{array}{ccccc}
1 & 0 & 0 & 0 & 1 \\
0 & 1 & 0 & 0 & 1 \\
0 & 0 & 1 & 0 & 1 \\
0 & 0 & 0 & 1 & 1 \\
1 & 1 & 1 & 1 & 0
\end{array}\right)
$$

with $\operatorname{rank} R_{F_{0}}=5$ and $\operatorname{rank} R_{F_{1}}=4$, so $r_{4}\left(\mathcal{C} l_{F_{0}}\right)=r_{4}\left(\mathcal{C} l_{F_{1}}\right)=0$. Hence $F_{0}, F_{1} \in \bar{X}_{4,0 ; n}^{*}$ and $\mathcal{S}_{0}(N) \cup \mathcal{S}_{1}(N) \subset \bar{X}_{4,0 ; n}^{*}$, so we have

$$
\delta_{4,0}^{*} \geq \lim _{\substack{n \rightarrow \infty \\ n: \text { odd }}} \frac{\left|\mathcal{S}_{0}(N)\right|}{\left|\bar{X}_{4 ; n}\right|}+\lim _{\substack{n \rightarrow \infty \\ n: \text { odd }}} \frac{\left|\mathcal{S}_{1}(N)\right|}{\left|\bar{X}_{4 ; n}\right|}=2^{-14}
$$

- Case $\left(\frac{P_{5}}{P_{i}}\right)=1$ for $1 \leq i \leq s<4$ and $\left(\frac{P_{5}}{P_{i}}\right)=-1$ for $s+1 \leq i \leq 4$. We have

$$
R_{F_{0}}=\left(\begin{array}{ll}
O_{1} & O_{2} \\
O_{3} & M_{0}
\end{array}\right), \quad R_{F_{1}}=\left(\begin{array}{cc}
O_{1} & O_{2} \\
O_{3} & M_{1}
\end{array}\right)
$$

where $O_{1}$ is the $s \times s$ zero matrix, $O_{2}$ is the $s \times(5-s)$ zero matrix, $O_{3}$ is the $(5-s) \times s$ zero matrix and $M_{0}, M_{1}$ are the $(5-s) \times(5-s)$ matrices given by

$$
M_{0}=\left(\begin{array}{ccccc}
1 & 0 & \cdots & 0 & 1 \\
0 & 1 & \cdots & 0 & 1 \\
\vdots & \vdots & & \vdots & \vdots \\
0 & 0 & \cdots & 1 & 1 \\
1 & 1 & \cdots & 1 & s+1
\end{array}\right), \quad M_{1}=\left(\begin{array}{ccccc}
1 & 0 & \cdots & 0 & 1 \\
0 & 1 & \cdots & 0 & 1 \\
\vdots & \vdots & & \vdots & \vdots \\
0 & 0 & \cdots & 1 & 1 \\
1 & 1 & \cdots & 1 & s
\end{array}\right)
$$


Since $\operatorname{rank} R_{F_{0}}=\operatorname{rank} M_{0}=5-s, \operatorname{rank} R_{F_{1}}=\operatorname{rank} M_{1}=4-s$, we have $r_{4}\left(F_{0}\right)=r_{4}\left(F_{1}\right)=s, F_{0}, F_{1} \in \bar{X}_{4, s ; n}^{*}$ and $\mathcal{S}_{0}(N) \cup \mathcal{S}_{1}(N) \subset \bar{X}_{4, s ; n}^{*}$. Hence

$$
\delta_{4, s}^{*} \geq \lim _{\substack{n \rightarrow \infty \\ n: \text { odd }}} \frac{\left|\mathcal{S}_{0}(N)\right|}{\left|\bar{X}_{4 ; n}\right|}+\lim _{\substack{n \rightarrow \infty \\ n: \text { odd }}} \frac{\left|\mathcal{S}_{1}(N)\right|}{\left|\bar{X}_{4 ; n}\right|}=2^{-14} .
$$

- Case $\left(\frac{P_{5}}{P_{i}}\right)=1$ for $1 \leq i \leq 4$. We have

$$
R_{F_{0}}=\left(\begin{array}{ccccc}
0 & 0 & 0 & 0 & 0 \\
0 & 0 & 0 & 0 & 0 \\
0 & 0 & 0 & 0 & 0 \\
0 & 0 & 0 & 0 & 0 \\
0 & 0 & 0 & 0 & 1
\end{array}\right), \quad R_{F_{0}}=\left(\begin{array}{ccccc}
0 & 0 & 0 & 0 & 0 \\
0 & 0 & 0 & 0 & 0 \\
0 & 0 & 0 & 0 & 0 \\
0 & 0 & 0 & 0 & 0 \\
0 & 0 & 0 & 0 & 0
\end{array}\right)
$$

with $\operatorname{rank} R_{F_{0}}=1$ and $\operatorname{rank} R_{F_{1}}=0$, so $r_{4}\left(\mathcal{C} l_{F_{0}}\right)=r_{4}\left(\mathcal{C} l_{F_{1}}\right)=4$. Hence $F_{0}, F_{1} \in \bar{X}_{4,4 ; n}^{*}$ and $\mathcal{S}_{0}(N) \cup \mathcal{S}_{1}(N) \subset \bar{X}_{4,4 ; n}^{*}$, so we have

$$
\delta_{4,4}^{*} \geq \lim _{\substack{n \rightarrow \infty \\ n: \text { odd }}} \frac{\left|\mathcal{S}_{0}(N)\right|}{\left|\bar{X}_{4 ; n}\right|}+\lim _{\substack{n \rightarrow \infty \\ n: \text { odd }}} \frac{\left|\mathcal{S}_{1}(N)\right|}{\left|\bar{X}_{4 ; n}\right|}=2^{-14} .
$$

\section{References}

[1] S. Bae, S. Hu, and H. Jung, The generalized Rédei matrix for function fields, Finite Fields Appl. 18 (2012), no. 4, 760-780.

[2] F. Gerth, Quadratic fields with infinite Hilbert 2-class field towers, Acta. Arith. 106 (2003), no. 2, 151-158.

[3] F. Hajir, On a theorem of Koch, Pacific J. Math. 176 (1996), no. 1, 15-18.

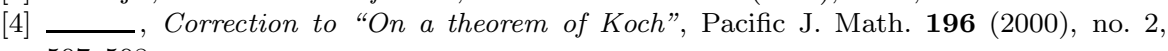
$507-508$.

[5] H. Koch, Über den 2-Klassenkörperturm eines quadratischen Zahlkörpers, J. Reine Angew. Math. 214/215 (1964), 201-206.

[6] J. Martinet, Tours de corps de classes et estimations de discriminants, Invent. Math. 44 (1978), no. 1, 65-73.

[7] M. Rosen, The Hilbert class field in function fields, Exposition. Math. 5 (1987), no. 4, $365-378$

[8] _ Number Theory in Function Fields, Graduate Texts in Mathematics, 210. Springer-Verlag, New York, 2002.

[9] R. Schoof, Algebraic curves over $\mathbb{F}_{2}$ with many rational points, J. Number Theory 41 (1992), no. 1, 6-14.

[10] C. Wittmann, Densities for 4-ranks of quadratic function fields, J. Number Theory 129 (2009), no. 10, 2635-2645.

Department of Mathematics Education

Chungbuk National University

Cheonguu 361-763, Korea

E-mail address: hyjung@chungbuk.ac.kr 